\title{
Association of Per3 length polymorphism with bipolar I disorder and schizophrenia
}

This article was published in the following Dove Press journal:

Neuropsychiatric Disease and Treatment

9 December 2014

Number of times this article has been viewed

Ramanujam Karthikeyan'

Chellamuthu

Ramasubramanian ${ }^{2}$

Gautham Arunachal'

Ahmed S BaHammam ${ }^{3}$

David Warren Spence ${ }^{4}$

Daniel P Cardinali ${ }^{5}$

Gregory M Brown ${ }^{6}$

Seithikurippu R Pandi-

Perumal $^{7}$

'Department of Animal Behaviour and Physiology, School of Biological Sciences, Madurai Kamaraj University, Madurai, India; ${ }^{2}$ MS Chellamuthu Trust and Research Foundation, KK Nagar, Madurai, India; ${ }^{3}$ University Sleep Disorders Center, College of Medicine, National Plan for Science and Technology, King Saud University, Riyadh, Saudi Arabia; ${ }^{4}$ Independent researcher, Toronto, Ontario,

Canada; ${ }^{5}$ Department of Teaching and Research, Faculty of Medical Sciences, Pontificia Universidad Católica Argentina, Buenos Aires, Argentina; ${ }^{6}$ Centre for Addiction and Mental Health, University of Toronto, Toronto, Ontario, Canada; ${ }^{7}$ Center for Healthful Behavior Change (CHBC), Division of Health and Behavior, Department of Population Health, NYU Langone Medical Center, Clinical and Translational Research Institute, New York, New York, USA

Correspondence: Ganapathy Marimuthu Department of Animal Behaviour and Physiology, School of Biological Sciences, Madurai Kamaraj University, Madurai 625 02I, Tamil Nadu, India

$\mathrm{Tel}+91979$ I696463

Fax +9| 452 2459/8I

Email emailboxgm@gmail.com
Background: Sleep-wake disturbances have frequently been reported in bipolar disorder and schizophrenia, and are considered to be caused by an underlying circadian rhythm disorder. The study presented here was designed to investigate the existence of Per3 polymorphism in bipolar disorder type I (BD-I) and schizophrenic patients in South India.

Methods: Blood samples were collected from 311 BD-I patients, 293 schizophrenia patients, and 346 age- and sex-matched normal controls. Per3 genotyping was performed on DNA by polymerase chain reaction using specific primers.

Results: An increased prevalence of five repeat homozygotes was seen in BD-I patients as compared with healthy controls (odds ratio $=1.72$ [95\% confidence interval: $1.08-2.76, P=0.02]$ ). In BD-I patients, the frequency of the five repeat allele was higher (allele frequency $=0.41$ ), and that of the four repeat allele lower (allele frequency $=0.36)\left(\chi^{2}=4.634 ; P<0.03\right)$ than in the control group. No significant association was observed in the allele frequencies of four and five repeat alleles in schizophrenia patients when compared with controls.

Conclusion: The occurrence of the five repeat allele of Per3 may be a risk factor for BD-I onset in this ethnic group.

Keywords: circadian rhythms, clock genes, Per3 polymorphism, bipolar disorder, schizophrenia

\section{Introduction}

Most organisms have evolved an internal timekeeping system as a temporal program for adapting to the rhythmic environmental fluctuations which accrue from the earth's rotation (eg, light-dark and temperature cycles). In humans this system involves, numerous behavioral and physiological parameters, including sleep-wake behavior and emotional and cognitive functioning, all of which oscillate rhythmically with a circadian pattern. ${ }^{1,2}$ There is a close interdependency between the circadian system and the body's homeostatic mechanisms. There is evidence suggesting that circadian processes, involving 24-hour oscillations in sleep propensity, interact with homeostatic mechanisms such as increases in sleep drive during wakefulness and reductions in drive during sleep. ${ }^{3,4}$ Consequently, the cross talk between these two systems is a reliable predictor of the sleep onset and offset of individuals. ${ }^{5,6}$

In certain psychiatric conditions, however, these rhythmicities may be disrupted. Sleep perturbations, reductions in sleep onset latency, early morning awakenings, and specifically disturbed rapid eye movement sleep have been observed in patients with the diagnosis of bipolar disorder (BD). ${ }^{7,8}$ Indeed, several reports have provided evidence suggesting that the desynchronization of the internal timekeeping system can be an important contributing factor in the onset of BD. ${ }^{9,10}$

Abnormal sleep-wake cycles, including changes in circadian phase (both advances and delays), discontinuous and compartmentalized sleep episodes, and disassembly 
in rest-activity cycles, have also been observed in patients suffering from schizophrenia. ${ }^{11-13}$

A variable tandem repeat polymorphism coding for 18 amino acids has been identified in exon 18 of Per3, a circadian clock gene involved in the regulation of the transcription-translation feedback loop generating 24-hour periodicity. ${ }^{14}$ This polymorphism has been linked to a variety of circadian and pathological phenomena. For example, there is evidence that Per3 length polymorphism is associated with sleep homeostasis and cognition, ${ }^{15}$ diurnal preference, ${ }^{16-18}$ and delayed sleep-phase syndrome. ${ }^{16,18}$

In one study, five repeat homozygotes of Per3 genotype were found to be associated with the onset of BD. ${ }^{19}$ By contrast, the postpartum onset of BD correlated with four repeat homozygotes. ${ }^{20}$ Nievergelt et $\mathrm{al}^{21}$ reported evidence for the association of Per3 haplotypes with BD, whereas in their replicative study they did not find any association between manic-depressive illness and $\operatorname{Per} 3$ repeat polymorphism. ${ }^{22}$ In schizophrenia, two single nucleotide polymorphisms were reported to be associated with the disorder ${ }^{9}$ but no association of a Per3 repeat polymorphism was found in 148 ethnically Han Chinese schizophrenics. ${ }^{23}$ In view of these conflicting reports, and to further clarify the role of Per3 in the etiology of BD and schizophrenia, we sought to investigate whether an association existed between Per3 length polymorphism and bipolar disorder type I (BD-I) or schizophrenia in a group of South Indian patients. To the best of our knowledge, this is the first study on this matter to have been carried out in an Indian population.

\section{Methods}

\section{Ethics statement}

All information with regard to the protocol was explained to the participants, and their written consent was obtained. The guidelines for the ethical treatment of patients in investigational studies were followed ${ }^{24}$ and the experimental protocol was reviewed and approved by the Institutional Ethical Committee of the Madurai Kamaraj University.

\section{Sample collection}

Blood samples $(2 \mathrm{~mL})$ were collected from patients suffering from BD-I $(n=311$, male $=48.9 \%$, female $=51.1 \%$ ) and schizophrenia $(\mathrm{n}=293$, male $=61.4 \%$, female $=38.6 \%)$, and normal controls $(n=346$, male $=53.2 \%$, female $=46.8 \%)$. The mean ages of participants in the three groups were $37.8 \pm 10.6$ years ( \pm standard deviation; BD-I patients), 36.4 \pm 10.1 years (schizophrenia patients), and $35.1 \pm 8.8$ years (normal controls). All BD-I and schizophrenia patients included in the study were either in- or outpatients of the MS Chellamuthu
Trust and Research Foundation, located in Madurai city. Patient and control information was collected over 3 years. Clinical interviews of the patients were undertaken using the Mini-International Neuropsychiatric Interview ${ }^{25}$ and clinical histories of longstanding symptoms were taken. Diagnoses of patients were made by a trained psychiatrist and confirmed by a senior psychiatrist. More details about the patients, including symptoms and mood profile, were obtained from family members and/or a close relative.

Diagnostic and Statistical Manual of Mental Disorders, 4th edition (DSM-IV) diagnostic criteria for schizophrenia included all subtypes (paranoid, disorganized, catatonic, undifferentiated, and residual); BD-I diagnostic criteria also included all six DSM-IV subtypes. ${ }^{26}$

All BD-I and schizophrenia patients were undergoing routine treatment for their respective disorders. Control subjects were initially screened and excluded from participation if they exhibited any symptoms of schizophrenia or mood disorders, or if they reported that any of their first-degree relatives had such symptoms. Using unstructured interviews, healthy control subjects were selected after a psychiatric screening. No prescribed questionnaire was used to determine any type of symptom in the controls. Hospital staff, students, and staff of the university were selected as controls. Selected controls did not have any history of head injury, sleep disorders, or any other major disorder which could cause a bias. All the healthy controls and patients confirmed that they were of South Indian ancestry.

\section{Per3 genotyping}

DNA was isolated from participants' blood samples using the phenol-chloroform method. A polymerase chain reaction (PCR) was performed using specific primers (5'-TGTCTTTTCATGTGCCCTTACTT-3' and 5'-TGTCTGGCATTGGAGTTTGA-3') as described elsewhere. ${ }^{27}$ The PCR reaction was carried out in $10 \mu \mathrm{L}$ tubes containing $1 \mu \mathrm{L}$ of $1 \times$ PCR reaction buffer, $0.5 \mu \mathrm{L}(0.5 \mathrm{mM})$ of deoxynucleotide triphosphates (dNTPs) mix, $1.5 \mu \mathrm{L}$ $(0.4 \mathrm{mM})$ forward/reverse primer mix, $0.05 \mu \mathrm{L}(0.025 \mathrm{U})$ Taq polymerase enzyme (Genet Bio Co Ltd, Daejeon, South Korea), $2 \mu \mathrm{L}$ (100 ng) of genomic DNA and $4.95 \mu \mathrm{L}$ of Milli-Q ${ }^{\circledR}$ water. The PCR amplification conditions included an initial step of $94^{\circ} \mathrm{C}$ for 5 minutes, 40 cycles of amplification $\left(94^{\circ} \mathrm{C}\right.$ for 30 seconds, $60.6^{\circ} \mathrm{C}$ for 30 seconds, $72^{\circ} \mathrm{C}$ for 30 seconds), and a final extension at $72^{\circ} \mathrm{C}$ for 5 minutes. The resultant PCR products showed three genotypes based on their DNA size, characterized as 1) $P e r 3^{5 / 5}$ homozygous allele (401 bp), 2) $\operatorname{Per}^{4 / 4}$ homozygous allele (347 bp), and 


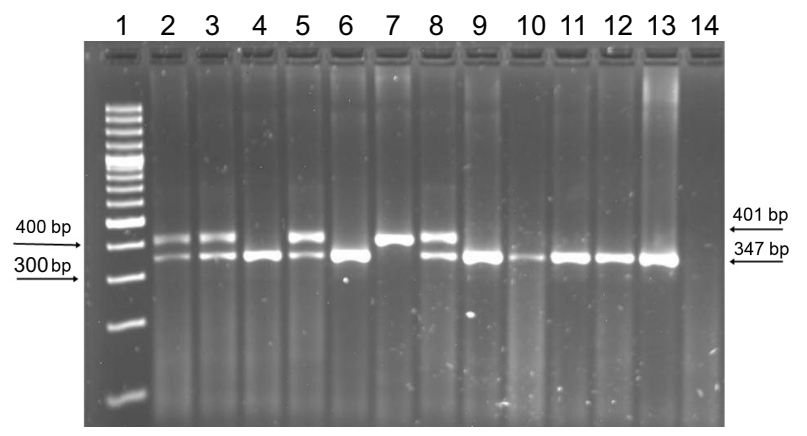

Figure I Agarose gel showing Per3 alleles.

Notes: Lane I: 100 bp marker; lanes 2, 3, 5, and 8: 4/5 heterozygous allele (40I and 347 bp); lanes 2-5: control samples; lanes 4, 6, and 9-13: 4/4 homozygous allele (347 bp); lanes 5-13: bipolar disorder patient samples; lane 7: 5/5 homozygous allele (40I bp); lane 14: no template control.

3) $P e r 3^{4 / 5}$ heterozygous allele (347 and $401 \mathrm{bp}$ ) (Figure 1). Subsequently, the amplified DNA sequences were sequenced for further confirmation.

\section{Statistical analysis}

The distributions of four and five repeat allele frequencies in the population were determined using the Hardy-Weinberg exact test. ${ }^{28}$ Deviations from Hardy-Weinberg equilibrium and the association of allele frequencies of four and five repeat alleles with BD-I, schizophrenia, and control subjects were analyzed by chi-square testing using SPSS software (v 20; IBM, Armonk, NY, USA). $P$-values of less than 0.05 were taken as evidence of statistical significance.

\section{Results}

Biallelic repeat variants of Per3 in BD-I, schizophrenia, and control subjects are summarized in Table 1. The distribution of Per3 genotypes in BD-I, schizophrenia, and normal healthy individuals were within the limits of the Hardy-Weinberg equilibrium. The observed and expected allele frequencies of both alleles in the three groups were similar and no group varied from the limit. The observed genotype frequencies of the controls were $4 / 4=141,4 / 5=163$, and $5 / 5=42$, and expected genotype frequencies were $4 / 4=143.1,4 / 5=158.8$, and $5 / 5=44.1$, and they were similar and did not show any statistical significance $(P>0.05)$. Similarly, the observed genotype frequencies of BD-I patients were $4 / 4=109,4 / 5=146$, and $5 / 5=56$ and their expected genotype frequencies did not have any major difference $(4 / 4=106.5,4 / 5=150.9$, and $5 / 5=53.5)$ and did not exhibit statistical significance $(P>0.05)$. Likewise, the observed $(4 / 4=115,4 / 5=137$, and $5 / 5=41)$ and expected genotype frequencies $(4 / 4=114.9,4 / 5=137.2$, and $5 / 5=40.9$ ) of the schizophrenia patients were similar and did not possess any significant difference $(P>0.05)$.

An increased prevalence of five repeat homozygotes was seen in BD-I patients (odds ratio $=1.72[95 \%$ confidence interval: $1.08-2.76, P=0.02])$. The frequency of five repeat allele (allele frequency $[\mathrm{AF}]=0.41$ ) was higher, and that of four repeat allele lower $(\mathrm{AF}=0.36)$, in $\mathrm{BD}-\mathrm{I}$ patients $\left(\chi^{2}=4.634\right.$, $P<0.03$ ) than in the control cohort (Table 1). The analysis of allele frequencies of four and five repeats did not reveal any statistically significant differences between patients with schizophrenia and the control group $\left(\chi^{2}=0.385 ; P=0.53\right)$.

There were differences in the allele frequencies of Per 3 and this observation was particularly notable among female patients with BD-I $\left(\chi^{2}=4.532 ; P<0.05\right)$ (Table 2$)$. No significant differences were found in the allele frequencies of four and five repeat alleles in male and female schizophrenia patients when compared with male and female control groups (Table 3 ).

\section{Discussion}

The foregoing results support the conclusion that Per3 polymorphism is associated with BD-I but not with schizophrenia. Benedetti et al $^{19}$ reported an association between earlier onset of BD with five repeat allele in the homozygous state. Another study suggested an influence of Per3 polymorphism in the postpartum onset of $\mathrm{BD} ;{ }^{20}$ interestingly, in the latter investigation, ${ }^{20}$ four repeat homozygotes have been associated with bipolar episodes. However, no significant difference was observed in the onset and number of episodes between Per 3 repeat alleles and BD. ${ }^{27}$

In a previous study, which used the Temperament and Character Inventory, ${ }^{29}$ five repeat homozygotes were found to be significantly linked with certain mood behaviors

Table I Genotypic distribution of the 4-/5-length polymorphism of Per3 in bipolar disorder type I (BD-I) and schizophrenia patients and controls

\begin{tabular}{|c|c|c|c|c|c|c|c|c|}
\hline \multirow[t]{2}{*}{ Group } & \multirow[t]{2}{*}{$\mathbf{N}$} & \multicolumn{3}{|c|}{ Genotype, n (\%) } & \multicolumn{2}{|c|}{ Allele, n (\%) } & \multirow[t]{2}{*}{$\chi^{2}$} & \multirow[t]{2}{*}{$P$} \\
\hline & & 4-/4- & $4-/ 5-$ & $5-/ 5-$ & 4- & 5- & & \\
\hline Control & 346 & I4I (40.8) & $163(47.1)$ & $42(12.1)$ & $445(0.64)$ & $247(0.36)$ & 4.634 & $0.03 *$ \\
\hline BD-I & 311 & $109(35.1)$ & I $46(47.0)$ & $56(18.0)$ & $364(0.59)$ & $258(0.4 I)$ & & \\
\hline Schizophrenia & 293 & $115(39.3)$ & $137(46.7)$ & $4 \mid(14.0)$ & $367(0.63)$ & $219(0.37)$ & 0.385 & 0.53 \\
\hline
\end{tabular}

Notes: 4-, 4-repeat allele; 5-, 5-repeat allele; *statistically significant.

Abbreviations: $\mathrm{N}$, number of samples; $\mathrm{n}$, number of genotypes and alleles. 
Table 2 Genotypic distribution of the 4-/5-length polymorphism of Per3 in bipolar disorder (BD) type I patients and controls based on sex

\begin{tabular}{|c|c|c|c|c|c|c|c|c|}
\hline \multirow[t]{2}{*}{ Group } & \multirow[t]{2}{*}{$\mathbf{N}$} & \multicolumn{3}{|c|}{ Genotype, n (\%) } & \multicolumn{2}{|c|}{ Allele, n (\%) } & \multirow[t]{2}{*}{$\chi^{2}$} & \multirow[t]{2}{*}{$P$} \\
\hline & & 4-/4- & 4-/5- & $5-/ 5-$ & 4- & $5-$ & & \\
\hline Male control & 184 & $73(39.7)$ & 90 (48.9) & $21(11.4)$ & $236(0.64)$ & $132(0.36)$ & 0.763 & 0.38 \\
\hline Male BD & 152 & $60(39.5)$ & $65(42.8)$ & $27(17.8)$ & $185(0.61)$ & $119(0.39)$ & & \\
\hline Female control & 162 & 68 (4I.9) & $73(45.1)$ & $21(12.9)$ & $209(0.65)$ & II $5(0.35)$ & 4.532 & $0.03 *$ \\
\hline Female BD & 159 & $49(30.8)$ & $8 \mid(50.9)$ & $29(18.3)$ & $179(0.56)$ & $139(0.44)$ & & \\
\hline
\end{tabular}

Notes: 4-, 4-repeat allele; 5-, 5-repeat allele; *statistically significant.

Abbreviations: $\mathrm{N}$, number of samples; $\mathrm{n}$, number of genotypes and alleles.

including novelty seeking, extravagance, cooperativeness, compassion, and integrated conscience in a subset of bipolar individuals. ${ }^{27}$ The genotype frequencies observed in the BD-I group in the present study (Per $3^{4 / 4}$ homozygous allele $=35.0 \%$, Per $^{4 / 5}$ heterozygous allele $=46.9 \%$, and Per $3^{5 / 5}$ homozygous allele $=18.0 \%$ ) were closely similar to those reported previously in $\mathrm{BD}$ patients $(35.3 \%, 46.5 \%$, and $18.2 \%$, respectively). ${ }^{19}$

Control allele frequencies of four repeat $(\mathrm{AF}=0.64)$ was slightly higher than the allele frequency found in Hindus of Maharashtra, India (AF $=0.57$ ) (M Thomas, Department of Biology, University College London, personal communication, March, 2012) and slightly less than the mean of the global population $(\mathrm{AF}=0.68) .{ }^{30}$ As indicated by post-Hardy-Weinberg equilibrium testing, the genotype and allele frequencies in the three groups presumably remained undisturbed by selection force. Furthermore, no selective advantage was observed over any particular Per 3 allele globally. ${ }^{30}$ In sex-based analysis, differences in the allele frequencies of both male and female BD-I patients were observed when compared with control subjects. Although the sample size was small, the differences between the observed and expected allele frequencies of the female BD-I group were found to be statistically significant, whereas these differences did not reach significance in the male BD-I group.

Since the frequency of occurrence of the five repeat allele was significantly greater in BD-I patients than in control individuals, the high occurrence of five repeat allele frequency may be correlated with the behavioral phenotype of the corresponding allele. For instance, patients with five repeat homozygotes showed short sleep latencies and spent more time in slow wave sleep. ${ }^{15}$ Five repeat allele individuals also exhibited more depressive symptoms and a lower motivational level than those with a four repeat allele. ${ }^{31}$ Sleep-wake behaviors such as a short sleep duration, early bedtime and sleep onset, spending more time in bed, hypervigilance, and reduced daytime sleep durations have been observed more frequently in subjects with five repeat homozygotes than in those with four repeat homozygotes or heterozygotes. ${ }^{32}$

How the Per3 gene contributes to the pathogenesis of psychiatric disorders is still unclear. A possible explanation for the occurrence of mood disorders is that in affected individuals, the circadian clock may show poor adaptability to different seasons. ${ }^{33,34}$ Another possible way in which a polymorphism of Per 3 could influence mood is by affecting sleep. ${ }^{33,35}$ No previous study has shown evidence specifically linking the Per3 genotype to any mental health outcome measures. ${ }^{32}$ The evidence presented in the current study is consistent with the hypothesis that Per3 alleles could influence the mental health status of individuals by modulating the sleep phase. Relevant to this suggestion has been the finding of an association between a single nucleotide polymorphism of Per 3 and poor sleep quality in $\mathrm{BD}$ patients. ${ }^{36}$ Morningness and eveningness preferences, which are strongly correlated with Per3, could modify the phase of the sleep and could conceivably result in abnormal mood profiles. In addition, Per3 polymorphism might perturb the delicate balance between mood and sleep and the balance toward episodes of mania or depression in individuals who have a high genetic

Table 3 Genotypic distribution of the 4-/5-length polymorphism of Per3 in schizophrenia patients and controls based on sex

\begin{tabular}{|c|c|c|c|c|c|c|c|c|}
\hline \multirow[t]{2}{*}{ Group } & \multirow[t]{2}{*}{$\mathbf{N}$} & \multicolumn{3}{|c|}{ Genotype, n (\%) } & \multicolumn{2}{|c|}{ Allele, n (\%) } & \multirow[t]{2}{*}{$\chi^{2}$} & \multirow[t]{2}{*}{$P$} \\
\hline & & 4-/4- & 4-/5- & $5-/ 5-$ & 4- & 5- & & \\
\hline Male control & 184 & $73(39.7)$ & $90(49)$ & $2 I(1 I .3)$ & $236(0.64)$ & $132(0.36)$ & 0.373 & 0.54 \\
\hline Male schizophrenia & 180 & $68(37.8)$ & $87(48.3)$ & $25(13.9)$ & $223(0.62)$ & $137(0.38)$ & & \\
\hline Female control & 162 & $68(42.0)$ & $73(45)$ & $2 I(13.0)$ & $209(0.65)$ & II $5(0.35)$ & 0.036 & 0.84 \\
\hline Female schizophrenia & 113 & $47(4 \mid .6)$ & $50(44.3)$ & $16(14.1)$ & I $44(0.64)$ & $82(0.36)$ & & \\
\hline
\end{tabular}

Notes: 4-, 4-repeat allele; 5-, 5-repeat allele.

Abbreviations: $N$, number of samples; $n$, number of genotypes and alleles. 
loading toward BD. This suggestion is consistent with previous findings that certain genotypes and alleles are more vulnerable to schedule changes and that this interaction can have behavioral and health-related consequences. ${ }^{37}$

The foregoing results in schizophrenic patients are in accordance with an earlier report that showed no correlation between $\operatorname{Per} 3$ repeat polymorphism and schizophrenia in a Chinese sample. ${ }^{23}$ Indeed, the onset of schizophrenia was not correlated with Per3 or the Clock gene variant, although a significant association of Clock allele frequency was found in a schizophrenic sample. ${ }^{23}$ It should be noted that elevated levels of interleukin- 6 have been observed in individuals affected with schizophrenia and a correlation of Per 3 repeat alleles with interleukin- 6 concentration was reported. ${ }^{38}$ These observations underscore the necessity for additional studies in larger samples and of different ethnic composition to further substantiate the conclusion that a lack of association between Per3 repeat alleles and schizophrenia truly exists.

Prevalence of Per3 repeat variants is hypothesized to augment phosphorylation of PER (PERIOD) proteins. Polymorphic repeats consist of sites for phosphorylation and a differential level of phosphorylation is caused by the insertion of an additional repeat. ${ }^{16}$ The present findings of an association of Per3 polymorphism with BD-I, but not with schizophrenia, are consistent with models suggesting that these represent different genres of psychiatric disorders. The findings further suggest that clock genes are complexly involved in either the expression and/or maintenance of these disorders. Furthermore, changes observed in the circadian phase of schizophrenia are quite distinct from the phase pattern of BD. ${ }^{9}$ This implies that the symptoms and pathogenesis of schizophrenia and BD are different and have a specific relationship with the circadian clock.

The study presented here suffered from several limitations. India comprises multi-ethnic groups and the possible impact of population stratification in genetic association studies must be taken into consideration. ${ }^{39}$ Population stratification raises the necessity for its confirmation in larger samples of patients and in different ethnic populations of the Indian subcontinent. Indeed, major genetic studies in the world have been carried out without considering the large Indian population and its different ethnicities. ${ }^{40}$ Since the controls were recruited from among hospital staff and students, an important bias could be that these subjects had higher levels of education and cognitive performance than patients. In addition, the present study gives no information on the association of the onset of BD-I among patients with the genotypic variants of Per3. Such a study is needed to support a direct link of the polymorphic variants observed with BD. Another disadvantage of the study is that it does not analyze the association between Per3 polymorphism and specific sleep behavior in the same population.

In any event, the data presented are compatible with the hypothesis that a length polymorphism in the circadian clock gene Per 3 influences the pathogenesis of BD-I but not of schizophrenia.

\section{Acknowledgments}

The authors sincerely thank Rukmani Manoharan, Raja, M Stalin Raja, S Tamilselvi, N Arul MuthuKumaran, Leishmann John Thumburaj, Ashok, and T Selvaraj for their help and assistance. The authors sincerely acknowledge the financial support received from various programs including DSTPURSE; UGC-CAS; NRCBS; UGC-Genomics Program; ANPCYT, Argentina (PICT 2012-0984); and the National Plan for Science and Technology program, King Saud University Project, Saudi Arabia. R Karthikeyan acknowledges the UGC for providing a Meritorious fellowship to him. G Marimuthu thanks INSA for a Senior Scientist position. The funders had no role in the study design, data collection and analysis, decision to publish, or preparation of the paper. Daniel P Cardinali is a senior investigator with CONICET, Argentina. The authors would like to thank the anonymous reviewers for their helpful and constructive comments, which greatly contributed to improving the final version of the paper. They would also like to thank the Editor for his generous comments and support during the review process.

\section{Disclosure}

All authors declare that they have no proprietary, financial, professional, nor any other personal interest of any nature or kind in any product or services and/or company that could be construed or considered to be a potential conflict of interest that might have influenced the views expressed in this paper.

\section{References}

1. Golombek DA, Casiraghi LP, Agostino PV, et al. The times they're a-changing: effects of circadian desynchronization on physiology and disease. J Physiol Paris. 2013;107(4):310-322.

2. Meijer JH, Michel S, Vanderleest HT, Rohling JH. Daily and seasonal adaptation of the circadian clock requires plasticity of the SCN neuronal network. Eur J Neurosci. 2010;32(12):2143-2151.

3. Franken P, Dijk DJ. Circadian clock genes and sleep homeostasis. Eur J Neurosci. 2009;29(9):1820-1829.

4. Moszczynski A, Murray BJ. Neurobiological aspects of sleep physiology. Neurol Clin. 2012;30(4):963-985.

5. Albrecht U. Circadian clocks and mood-related behaviors. Handb Exp Pharmacol. 2013;(217):227-239. 
6. Bersani FS, Iannitelli A, Pacitti F, Bersani G. Sleep and biorythm disturbances in schizophrenia, mood and anxiety disorders: a review. Riv Psichiatr. 2012;47(5):365-375.

7. Lauterbach D, Behnke C, McSweeney LB. Sleep problems among persons with a lifetime history of posttraumatic stress disorder alone and in combination with a lifetime history of other psychiatric disorders: a replication and extension. Compr Psychiatry. 2011;52(6):580-586.

8. Boivin DB. Influence of sleep-wake and circadian rhythm disturbances in psychiatric disorders. J Psychiatry Neurosci. 2000;25(5):446-458.

9. Mansour HA, Wood J, Logue T, et al. Association study of eight circadian genes with bipolar I disorder, schizoaffective disorder and schizophrenia. Genes Brain Behav. 2006;5(2):150-157.

10. Bunney JN, Potkin SG. Circadian abnormalities, molecular clock genes and chronobiological treatments in depression. Br Med Bull. 2008;86:23-32.

11. Wulff K, Dijk DJ, Middleton B, Foster RG, Joyce EM. Sleep and circadian rhythm disruption in schizophrenia. Br J Psychiatry. 2012; 200(4):308-316.

12. Monti JM, BaHammam AS, Pandi-Perumal SR, et al. Sleep and circadian rhythm dysregulation in schizophrenia. Prog Neuropsychopharmacol Biol Psychiatry. 2013;43:209-216.

13. Gottesmann C. [Dreaming and schizophrenia: a common neurobiological background?]. Med Sci (Paris). 2006;22(2):201-205. French.

14. Ebisawa T, Uchiyama M, Kajimura N, et al. Association of structural polymorphisms in the human period 3 gene with delayed sleep phase syndrome. EMBO Rep. 2001;2(4):342-346.

15. Viola AU, Archer SN, James LM, et al. PER3 polymorphism predicts sleep structure and waking performance. Curr Biol. 2007;17(7): 613-618.

16. Archer SN, Robilliard DL, Skene DJ, et al. A length polymorphism in the circadian clock gene Per3 is linked to delayed sleep phase syndrome and extreme diurnal preference. Sleep. 2003;26(4):413-415.

17. Ellis J, von SM, Jones KH, Archer SN. Association between specific diurnal preference questionnaire items and PER3 VNTR genotype. Chronobiol Int. 2009;26(3):464-473.

18. Pereira DS, Tufik S, Louzada FM, et al. Association of the length polymorphism in the human Per3 gene with the delayed sleep-phase syndrome: does latitude have an influence upon it? Sleep. 2005;28(1): 29-32.

19. Benedetti F, Dallaspezia S, Colombo C, Pirovano A, Marino E, Smeraldi E. A length polymorphism in the circadian clock gene Per3 influences age at onset of bipolar disorder. Neurosci Lett. 2008;445(2): 184-187.

20. Dallaspezia S, Lorenzi C, Pirovano A, Colombo C, Smeraldi E, Benedetti F. Circadian clock gene Per3 variants influence the postpartum onset of bipolar disorder. Eur Psychiatry. 2011;26(3):138-140.

21. Nievergelt CM, Kripke DF, Barrett TB, et al. Suggestive evidence for association of the circadian genes PERIOD3 and ARNTL with bipolar disorder. Am J Med Genet B Neuropsychiatr Genet. 2006;141B(3):234-241.

22. Kripke DF, Nievergelt CM, Joo E, Shekhtman T, Kelsoe JR. Circadian polymorphisms associated with affective disorders. J Circadian Rhythms. 2009;7:2.

23. Zhang J, Liao G, Liu C, et al. The association of CLOCK gene T3111C polymorphism and hPER3 gene 54-nucleotide repeat polymorphism with Chinese Han people schizophrenics. Mol Biol Rep. 2011; 38(1):349-354.
24. Portaluppi F, Smolensky MH, Touitou Y. Ethics and methods for biological rhythm research on animals and human beings. Chronobiol Int. 2010;27(9-10):1911-1929.

25. Sheehan DV, Lecrubier Y, Sheehan KH, et al. The Mini-International Neuropsychiatric Interview (M.I.N.I.): the development and validation of a structured diagnostic psychiatric interview for DSM-IV and ICD-10. J Clin Psychiatry. 1998;59 Suppl 20:22-33.

26. American Psychiatric Association (APA). Diagnostic and Statistical Manual of Mental Disorders. 4th ed. Arlington, VA: APA; 1994.

27. Artioli P, Lorenzi C, Pirovano A, et al. How do genes exert their role? Period 3 gene variants and possible influences on mood disorder phenotypes. Eur Neuropsychopharmacol. 2007;17(9):587-594.

28. Rodriguez S, Gaunt TR, Day IN. Hardy-Weinberg equilibrium testing of biological ascertainment for Mendelian randomization studies. Am J Epidemiol. 2009;169(4):505-514.

29. Cloninger CR, Przybeck TR, Svrakic DM, Wetzel RD. The Temperament and Character Inventory (TCI): A Guide to its Development and Use. St Louis, MO: Center for Psychobiology of Personality, Washington University; 1994.

30. Nadkarni NA, Weale ME, von SM, Thomas MG. Evolution of a length polymorphism in the human PER3 gene, a component of the circadian system. J Biol Rhythms. 2005;20(6):490-499.

31. Guess J, Burch JB, Ogoussan K, et al. Circadian disruption, Per3, and human cytokine secretion. Integr Cancer Ther. 2009;8(4):329-336.

32. Lázár AS, Slak A, Lo JC, et al. Sleep, diurnal preference, health, and psychological well-being: a prospective single-allelic-variation study. Chronobiol Int. 2012;29(2):131-146.

33. Kripke DF, Mullaney DJ, Atkinson M, Wolf S. Circadian rhythm disorders in manic-depressives. Biol Psychiatry. 1978;13:335-351.

34. Bodenstein C, Gosak M, Schuster S, Marhl M, Perc M. Modeling the seasonal adaptation of circadian clocks by changes in the network structure of the suprachiasmatic nucleus. PLoS Comput Biol. 2012; 8(9):e1002697.

35. Archer SN, Carpen JD, Gibson M, et al. Polymorphism in the PER3 promoter associates with diurnal preference and delayed sleep phase disorder. Sleep. 2010;33(5):695-701.

36. Rocha PM, Neves FS, Alvarenga NB, Hughet RB, Barbosa IG, Corrêa H. Association of Per3 gene with bipolar disorder: comment on "Association study of 21 circadian genes with bipolar I disorder, schizoaffective disorder, and schizophrenia". Bipolar Disord. 2010;12(8): 875-876.

37. Gamble KL, Motsinger-Reif AA, Hida A, et al. Shift work in nurses: contribution of phenotypes and genotypes to adaptation. PLoS One. 2011;6(4):e18395.

38. Frommberger UH, Bauer J, Haselbauer P, Fräulin A, Riemann D, Berger M. Interleukin-6-(IL-6) plasma levels in depression and schizophrenia: comparison between the acute state and after remission. Eur Arch Psychiatry Clin Neurosci. 1997;247(4):228-233.

39. Indian Genome Variation Consortium. Genetic landscape of the people of India: a canvas for disease gene exploration. J Genet. 2008;87(1): $3-20$.

40. Rosenberg NA, Mahajan S, Gonzalez-Quevedo C, et al. Low levels of genetic divergence across geographically and linguistically diverse populations from India. PLoS Genet. 2006;2(12):e215.
Neuropsychiatric Disease and Treatment

\section{Publish your work in this journal}

Neuropsychiatric Disease and Treatment is an international, peerreviewed journal of clinical therapeutics and pharmacology focusing on concise rapid reporting of clinical or pre-clinical studies on a range of neuropsychiatric and neurological disorders. This journal is indexed on PubMed Central, the 'PsycINFO' database and CAS,

\section{Dovepress}

and is the official journal of The International Neuropsychiatric Association (INA). The manuscript management system is completely online and includes a very quick and fair peer-review system, which is all easy to use. Visit http://www.dovepress.com/testimonials.php to read real quotes from published authors. 